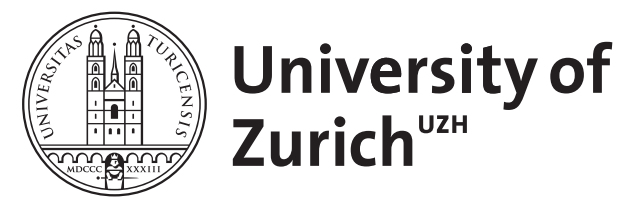

\title{
Targeting apoptosis pathways in glioblastoma
}

Eisele, G ; Weller, M

\begin{abstract}
The treatment of glioblastoma remains a major challenge for clinicians since these highly aggressive brain tumors are relatively resistant towards radio- and chemotherapy. The pathways that control apoptosis are altered in glioblastoma cells leading to resistance towards apoptotic stimuli in general. In this review we describe the alterations affecting the p53 pathway, the BCL-2 protein family, the inhibitor of apoptosis proteins and several growth factor pathways involved in the regulation of programmed cell death and define possible targets for new therapies within these apoptotic pathways in glioblastomas. Moreover, we review strategies to target death receptor pathways, most notably to render the glioblastoma cells more susceptible towards this approach without enhancing toxicity in general. Most of the strategies targeting apoptosis in glioblastomas presented here are in a pre-clinical stage of development, however, they all share the ultimative goal to improve the outcome for glioblastoma patients.
\end{abstract}

DOI: https://doi.org/10.1016/j.canlet.2010.12.012

Posted at the Zurich Open Repository and Archive, University of Zurich

ZORA URL: https://doi.org/10.5167/uzh-50500

Journal Article

Accepted Version

Originally published at:

Eisele, G; Weller, M (2013). Targeting apoptosis pathways in glioblastoma. Cancer Letters, 332(2):335345.

DOI: https://doi.org/10.1016/j.canlet.2010.12.012 


\section{RESPONSE}

Whole-brain radiotherapy for primary CNS lymphoma?

The G-PCNSL-SG-1 trial is the only completed randomized phase III trial in patients with primary CNS lymphoma (PCNSL) (1). It answered the question repeatedly identified in many review articles as the most challenging and important question to answer in the treatment of PCNSL: does the omission of whole brain radiotherapy (WBRT) from the treatment of newly diagnosed PCNSL compromise survival? The answer is no.

G-PCNSL-SG-1 was an investigator-initiated trial that was done without industry funding and designed in 1998. It should not be judged by quality criteria of present industry-sponsored trials. We detailed limitations in our Article and find no new considerations in the comments of Ferreri and colleagues.

(i) Although our eligibility criteria were much the same as in many smaller trials, we enrolled a patient population with a high median age (63 years [nearly a quarter over 70 years]) and low Karnofsky performance scores. We showed a complete-response rate of 32.4\% in 401 patients which compares favourably with $18 \%$ in 40 patients in a contemporary oligocentric trial by the International Extranodal Lymphoma Study Group (2).

(ii) By contrast to the latter study (2), we analysed a possible negative impact on outcome from centers with a small patient volume, but did not identify one, suggesting that decentralised care is not harmful to outcome.

(iii) We did not monitor neurotoxicity only by MRI, but also clinically, which does not differ from the technique used by many other investigators..

(iv) The statement that $40 \%$ of the patients were excluded from analysis is misleading, if not deliberately unfair. Randomisation was appropriately done up-front, before high-dose methotrexate-based chemotherapy, but could only become effective if patients were eligible 
for either consolidation or salvage. Patients would thus inevitably be lost during the intended six cycles of chemotherapy. Moreover, the second, not unexpected loss of patients occurred (a) at the step where randomisation led patients in complete remission to receive WBRT and (b) patients without complete remission to receive second-line chemotherapy instead of WBRT. Such a randomisation is difficult to perform, but is the only way to address the value of WBRT. We assume that later randomization after completion of chemotherapy would have made randomization even more difficult. Yet, when all patients included $(\mathrm{n}=551)$ and all patients meeting eligibility criteria who were started on primary chemotherapy $(n=526)$ were analysed for outcome, similar results for the primary endpoint as in the PP and ITT populations were found, indicating that the study results were not biased by patient selection. Of 411 ITT patients at the beginning of the randomised intervention, 318 (77\%) were included in the PP analysis.

(v) We would like to thank our colleagues for pointing out one of our major findings: WBRT can be postponed until relapse without compromising survival. Yet, it is trivial that options for treatment at first recurrence differed between study arms in our trial: patients who received chemotherapy alone as their first-line therapy often had WBRT later on whereas already irradiated patients did not receive a second course of WBRT. The salvage chemotherapy protocols were similar in both arms.

In conclusion, G-PCNSL-SG-1 proves that randomized phase III trials with an adequate number of patients are possible in this rare disease. It has set a standard of clinical trial design and conduct in PCNSL. Ferreri and colleagues define this standard as low here. We encourage them to join forces and show as early as possible that improved trials with more definitive answers can be undertaken. Time will tell.

Agnieszka Korfel, Peter Martus, Eckhard Thiel, Berlin, Germany

Michael Weller, Zurich, Switzerland, 
For the German PCNSL Study Group

References

Thiel E, Korfel A, Martus P, et al. G-PCNSL-SG-1 randomised phase III trial of high-dose methotrexate with or without whole brain radiotherapy for primary central nervous system lymphoma. Lancet Oncol 2010;11:1036-47.

Ferreri AM, Reni M, Foppoli M, et al. High-dose cytarabine plus higy-dose methotrexate versus high-dose methotrexate alone in patients with primary CNS lymphoma: a randomised phase 2 trial. Lancet 2009;374:1512-20. 